\title{
Atomically Precise Nanocrystals
}

\author{
Zeger Hens ${ }^{*}, \dagger,+$, Jonathan De Roo*,§ \\ †Physics and Chemistry of Nanostructures, Ghent University, 90oo Ghent, Belgium \\ *Center for Nano and Biophotonics, Ghent University, 9000 Ghent, Belgium \\ $\S$ Department of chemistry, University of Basel, 4058 Basel, Switzerland
}

\begin{abstract}
Nanocrystals are a state-of-matter in the border area between molecules and bulk materials. Unlike bulk materials, nanocrystals have size-dependent properties, yet the question remains whether nanocrystal properties can be analyzed, understood, and controlled with atomic precision, a key characteristic of molecules. Acknowledging the inclination of nanocrystals to form defect structures, we first outline the prospects of atomically precise analysis. A broad spectrum of analytical methods has become available over the last five years, such that for almost any nanocrystal, atomically precise average structures can be determined to represent a polydisperse ensemble and explore structureproperty relations. Atomically precise synthesis, on the other hand, remains an outstanding challenge that may well face fundamental limitations. However, to amplify properties and prime nanocrystals for specific applications, full atomic precision may not be needed. Examples of an atomic precision light approach, focusing on exact thickness or facet control, exist and can inspire scientists to explore atomic precision in nanocrystal research further.
\end{abstract}

\section{INTRODUCTION}

The scientific interest in nanocrystals has been increasing continuously over the last 25 years. Interestingly, the more common wording nanoparticle first appeared in the context of drug delivery systems. ${ }^{1}$ In contrast, the term nanocrystal was introduced to describe materials with nanocrystalline grains, which were put forward as a novel state of matter. ${ }^{2}$ While researchers now rather see a nanocrystal as an isolated, nanometer-sized crystallite, ${ }^{3}$ the idea of a new state of matter persisted. For such individual nanocrystallites, it was found that atoms are arranged in crystal structures, not unlike the corresponding bulk material, yet nanocrystal properties may be strongly size-dependent. 4 In this way, nanocrystals move beyond the idea that materials can be characterized by intensive properties that do not depend on the amount of material.

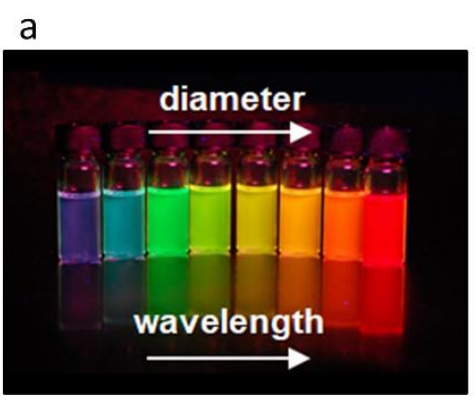

b

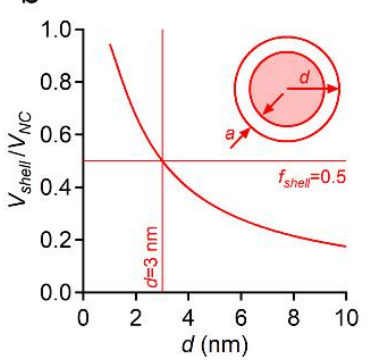

The size-dependence of material properties can have different origins. The change of the electronic structure of semiconductors with the nanocrystal size reflects the increased energy needed to confine electrons in smaller volumes.4-5 The stronger light absorption and tunable emission color induced by quantum confinement underpins the use of such nanocrystals as spectrum-ondemand semiconductors, 3 and have made size series of semiconductor nanocrystals a most tangible example of quantum mechanics in action (see Figure 1a). On the other hand, size-dependent physicochemical properties are often the result of the increased surface-to-volume ratio. With decreasing size, the contribution of the surface tension to the free energy of a crystallite becomes more important. ${ }^{4}$ Such surface effects can hardly be underestimated. Roughly half the atoms in a nanocrystal with a diameter of $3 \mathrm{~nm}$ will be surface atoms (see Figure
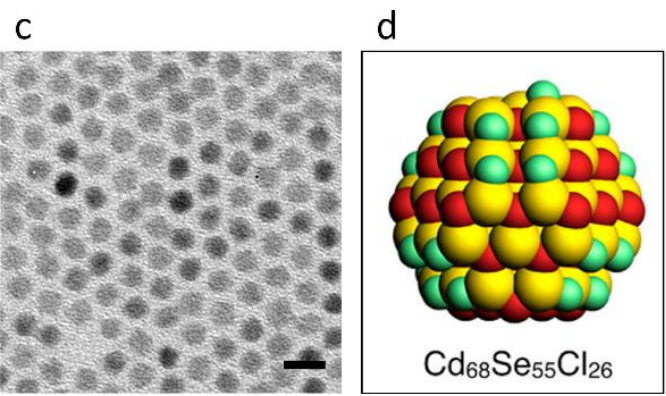

Figure 1. (a) The sequence of CdSe nanocrystal dispersions highlights the redshift of the emission wavelength with increasing nanocrystal diameter. (b) Variation of the fraction $f_{\text {shell }}$ of surface atoms as a function of the nanocrystal diameter. $f_{\text {shell }}$ is calculated as the shell vs. total nanocrystal volume using the depicted concentric sphere model with $a=0.31 \mathrm{~nm}$. (c) Transmission electron microscopy image of PbSe nanocrystals with a size dispersion of $5 \%$. The scale bar measures 10 nm. See ref 6 for experimental details. (d) Structure model of a CdSe nanocrystal terminated by chloride ligands with chemical formula as indicated. See ref 7 . 
1b). In that case, one single surface energy provides an inadequate description of the nanocrystal surface properties, which will depend strongly on the exposed facets, edges, and corners. This complexity creates ample opportunities for chemistry, including the introduction of functionality through adsorbates (ligands), the direct exploitation of nanocrystal surfaces for catalysis, ${ }^{8}$ or the combined approach of ligand-assisted catalysis. ${ }^{9}$

When size-dependence is the central theme, methods for precision synthesis are needed. Indeed, even if single nanocrystals can be studied by various techniques, analyzing nanocrystals one-by-one is often a low throughput endeavor, and attaining meaningful statistics can be difficult. Many investigations rely on nanocrystal ensembles, for which broad size distributions will cloak any size-dependent material property. The task is thus to produce monodisperse nanocrystal ensembles, and colloidal synthesis methods proved well suited. In some cases, size distributions with a relative standard deviation of just $5 \%$ have been obtained (see Figure 1c). ${ }^{6}$ Moreover, by carrying out reactions under kinetic control, ${ }^{10}$ one can produce nanocrystals with a variety of shapes, including rods, ${ }^{11-14}$ nanoplatelets, ${ }^{15-21}$ or tetrapods. ${ }^{22-23}$

It goes without saying that the progress in chemical synthesis profoundly helped scientific research into nanocrystals. But what about the next step? Can we imagine synthesizing nanocrystals with atomic precision? Would it be possible to produce ensembles of nanocrystals, each having the same chemical formula and chemical structure? Small molecules are defined by a single chemical formula and a unique chemical structure. The same holds true for clusters, such as $\mathrm{Au}_{55}\left(\mathrm{PPh}_{3}\right)_{12} \mathrm{Cl}_{6}$ or $\mathrm{In}_{37} \mathrm{P}_{20}\left(\mathrm{O}_{2} \mathrm{CCH}_{2} \mathrm{Ph}\right)_{51}{ }^{24-25}$ But what about nanocrystals? Atomically precise nanocrystal structures can be drawn and computed (see Figure id), but having them physically available is what can make a real difference. Thinking of useful physical and chemical properties, ensembles of identical nanocrystals would exhibit absorption and emission spectra with well-defined, narrow, and gradually tunable lines. Moreover, atomistic control could boost catalytic activity by maximizing specific surface sites and all-identical nanocrystals would be the ideal starting point for forming superstructures by nanocrystal assembly. From an analytical perspective, ensembles of atomically precise nanocrystals will facilitate researchers to map structure/property relations. Moreover, simulations on model systems that are true digital copies of the material at hand could put such relations to the test. In this way, not only static properties but also the dynamic behavior of nanocrystals could be predicted under a specific load, such as temperature, illumination, or exposure to chemicals. Such an approach has great potential benefits for nanocrystal science and technology.

In this perspective, we address the status of atomic precision in nanocrystal science. First, we highlight that attaining atomic precision with nanocrystals may well face intrinsic limits. The single lowest energy structure of a nanocrystal will always be in the balance with a multitude of higher energy defect structures, suggesting entropy will eventually counteract atomic precision. Researchers can accept these variations in structure and composition as a matter of fact in nanocrystal research, and shift their goals to atomically precise analysis. Single-particle studies provide atomically precise information, but also ensemble level methods can yield valuable insight. Especially when different methods of analysis are combined to obtain a comprehensive description of composition, structure and surface termination, an atomically precise structure of the average nanocrystal in a monodisperse ensemble may emerge. Alternatively, nanocrystal synthesis could rise to the challenge and develop methods to overcome defect sensitivity. Here, guidelines can come from the established synthesis of nanocrystals with an atomically precise thickness or well-defined crystal facets, or the formation of magic size clusters with ever-increasing dimensions. ${ }^{26}$

\section{LIMITS TO ATOMIC PRECISION}
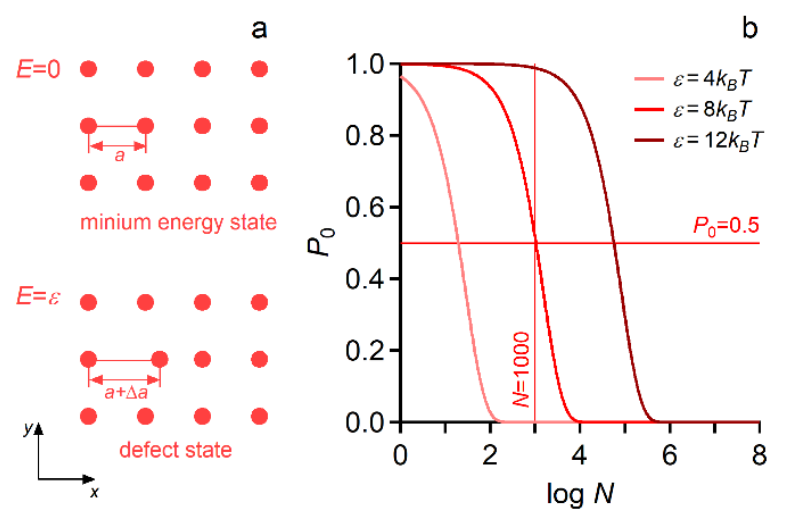

Figure 2. (a) Representation of a minimum energy structure of a $2 \mathrm{D}$ square lattice and a defect structure characterized by the displacement of a given atom. The defect state has an energy $\varepsilon$ above the minimum energy state. (b) The probability of finding the structure in the ground state as a function of the number of atoms $N$ in the lattice for different excess energies as indicated.

Opposite from the benzene molecule, where attempts to add a single hydrogen atom violate basic principles of chemical bonding, extending a CdSe nanocrystal with a single CdSe formula unit or a $\mathrm{Cd}$ oleate ligand sounds hardly problematic. So how realistic is atomic precision when talking about nanocrystals? To explore the limits to atomic precision, we start from the notion that a given nanocrystal has a unique minimum energy structure that is characterized by a specific average position of the constituting atoms. Structures with atoms in deviating positions will have higher energy, so we call these defect structures. Figure 2a shows the example of a square lattice for which the minimum energy state corresponds to an equal spacing $a$ between the atoms. In the same figure, we introduce an alternative state of that crystallite. This state has a point defect, represented by the displacement of an atom in the $+x$ direction, that has an energy $\varepsilon$ above the ground state energy. Figure $2 \mathrm{~b}$ represents the probability $P_{0}$ to find the nanocrystal in the minimum energy state as a function of the number $N$ of atoms in the nanocrystal, for different $\varepsilon$ values. Interestingly, one sees that a 1000 atom lattice has a $50 \%$ chance to develop point defects with an energy of $8 k_{B} T$. Moreover, while finding point defects with an energy of $4 k_{B} T$ is all but certain, higher energy defects should be nearly absent; an observation 
that underpins the idea that nanocrystals can be defectfree by self-purification. ${ }^{27-28}$

This simplified model highlights the trade-off between energy and entropy in nanocrystals, where the wide variety of possible defects quickly leads to deviations from the lowest energy structure. This tendency to form defect structures seems a pervasive property of nanocrystals. Due to their high formation energy, the concentration of Schottky defects - uncompensated vacancies - in metal nanocrystals is expected to decrease for crystallites smaller than $100 \mathrm{~nm},{ }^{29}$ yet in Bi nanocrystals, Schottky defects were still found to initiate melting upon heating. ${ }^{30}$ Similarly, simulations indicate that dislocation motion should no longer determine plastic deformation of nanometer-sized crystallites. ${ }^{3-32}$ However, texturing due to active dislocations was still observed in $3 \mathrm{~nm}$ nickel or gold crystals under high pressure. ${ }^{33-34}$ Wurtzite CdSe nanorods were found to exhibit multiple zinc blende stacking faults along the wurtzite $c$ axis. 35 Similarly, twin defects are a prominent feature in fcc gold nanocrystals, which result in nanocrystals featuring decahedral or icosahedral shapes.35-36 Even the ground state of $\mathrm{Au}_{55}$ clusters was predicted to consist of a manifold of nearly degenerate isomers. 37

The structure of colloidal nanocrystals is not limited to the stacking of atoms in the inorganic core. Metal, metal oxide, or semiconductor nanocrystals all feature outer facets terminated by ligands, either as a residue of the nanocrystal synthesis or as the result of deliberate surface functionalization. ${ }^{38}$ Continuing with the example of CdSe nanocrystals, adsorption sites on (100) facets exhibit mostly similar binding energies. ${ }^{39}$ Hence, as soon as not all binding sites are occupied, many, nearly equivalent distributions of ligands across a nanocrystal surface are possible, with a missing ligand acting as the point defect in the simplified model of Figure 2. Of particular relevance here is that ligand displacement is not necessarily a highenergy defect formation process. Specific complexing agents - often present during synthesis or purification can displace ligands in an overall exothermic process when the complexation energy compensates the ligand binding energy. $4^{4^{-}-41}$ Hence, the ligand shell of nanocrystals in an ensemble can readily differ by the distribution of ligands across the surface, but also by the number of ligands at the surface.

Figure $2 \mathrm{~b}$ highlights that the probability of finding $a$ nanocrystal in the lowest energy state strongly increases in the case of high energy point defects. Especially when considering doped nanocrystals, this statistical result led to the idea that nanocrystals expel impurities by selfpurification, ${ }^{27}$ especially when the doping energy were to increase with decreasing nanocrystal size. ${ }^{28}$ However, even if specific examples suggest self-purification to occur, ${ }^{42}$ the broad applicability of the concept remains a matter of debate. 43 Despite self-purification, the formation of doped nanocrystals proved possible by exploiting kinetic factors such as the adsorption of the dopant at the nanocrystal surface. ${ }^{44}$ Regarding the dopant as a defect, the latter point underscores the role of the synthesis method in the formation of defective nanocrystals. Especially under kinetic control, colloidal synthesis can be tweaked to produce nanocrystals with a variety of sizes, unusual shapes or composition, ${ }^{10}$ such as pentatwinned gold decahedrons ${ }^{45-46}$ or CdSe/CdS heteronanorods. ${ }^{47-48}$ Also metastable crystal structures, not attainable in the bulk become accessible. ${ }^{49-50}$ The flip side is that a given synthesis method may well produce nanocrystals with a distribution of kinetically-trapped metastable structures, instead of the single desired product.

In the above examples, both the core and the surface of nanocrystals appear very much defect prone, and eliminating defects by self-purification may not be the correct approach to atomic precision. Hence, even nanocrystal ensembles labeled monodisperse may readily feature a diversity of structures. Thinking of atomically precise nanocrystals, scientists should thus set their goals accordingly. The first option is to accept diversity as a given and shift the question to atomically precise analysis.

\section{ANALYSIS WITH ATOMIC PRECISION}

\section{Single crystal diffraction}

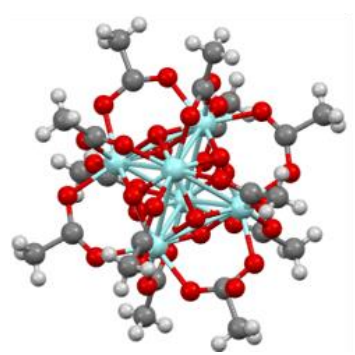

Graphene liquid cell TEM

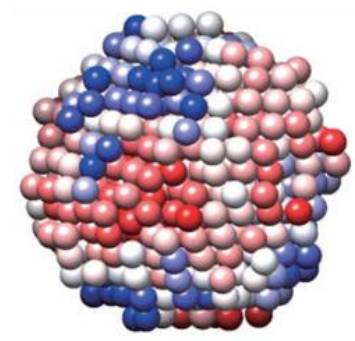

Figure 3. The atomically precise analysis result of either a small $\mathrm{Zr}_{6} \mathrm{O}_{4}(\mathrm{OH})_{4}\left(\mathrm{OOCCH}_{3}\right)_{12}$ cluster via single-crystal diffraction, constructed according to published data, ${ }^{1}$ or $\mathrm{Pt}$ nanocrystals via graphene liquid cell TEM..$^{2}$ Reprinted in part with permission from ref $5 \mathbf{2}$. Copyright 2020 The American Association for the Advancement of Science.

Single-crystal x-ray diffraction allows locating every atom except hydrogen in a structure with high precision. The method is widely used for the absolute structure determination of organic molecules and metal complexes and small clusters, see Figure 3. As it requires the growth of single crystals, the technique has mostly been applied to clusters with rigid ligands that assist the crystallization process, such as phenylacetate-terminated InP clusters with the structure formula $\operatorname{In}_{37} \mathrm{P}_{20}\left(\mathrm{O}_{2} \mathrm{CCH}_{2} \mathrm{Ph}\right)_{51} \cdot{ }^{25}$ Studies on gold clusters have further pushed single crystal diffraction towards larger objects. Using tertbutylbenzene thiol ligands, an entire sizes series was crystallized, from $\mathrm{Au}_{28}(1 \mathrm{~nm})$ to $\mathrm{Au}_{279}(2.2 \mathrm{~nm}) .53$ The latter can be considered a small nanocrystal. Given exact solutions for the structure, structure-function relationships could be established. While $A u_{28}-A_{133}$ clusters show molecular behavior, the $\mathrm{Au}_{279}$ cluster is metallic and features a plasmon resonance. 53 Despite this recent progress, single-crystal diffraction has important limitations for the structure analysis of nanocrystals. The ligands present the first problem. While colloidally stable nanocrystals are typically obtained with flexible or branched ligands, rigid ligands are typically required for crystallization and single crystal XRD. However, it is 
encouraging that, even with flexible ligands, nanocrystal superlattices can be formed, 54 and often even show alignment of their crystalline domains in 3 dimensions. This brings us one step closer to single crystal XRD, although the electron density of the ligands would be disordered. Furthermore, the unit cell of such a crystal would be several nanometers in size, causing the reflections to be compressed at very small angles. Therefore, measurements might happen in a similar fashion to protein crystallography, most likely at synchrotron facilities. However currently, the most existential problem for single crystal XRD on nanocrystals is the underlying polydispersity of the nanocrystals precludes the formation of true single crystal superlattices. This is a catch 22. The superstructure - and thus the nanocrystal building blocks - needs to be atomically precise for these building blocks to be determined with atomic precision. So what about the atomically precise analysis of single nanocrystals?

In the case of protein analysis, the difficulty of making sufficiently large single crystals inspired research into single (macro)molecule diffraction. Here, the use of fs pulsed $x$-rays can overcome the unavoidable issues of sample-damage that come with the use of higher intensity x-rays when imaging smaller structures. 55 Alternatively, electrons focused in a nanometer wide parallel beam in a transmission electron microscope (TEM) can be used to record diffraction patterns. In fact, structure analysis by $3 \mathrm{D}$ electron diffraction has become a rapidly developing technique that may find applications in nanocrystal research. ${ }^{5}$ Published examples, however, involve TEMbased imaging of single nanocrystals. For example, the structure of single Pt nanocrystals was determined by dispersing the crystallites in a graphene liquid cell, were the rotation freedom allows for taking consecutive images of the same nanocrystal under different orientations. ${ }^{57}$ Using a tomographic algorithm, the $3 \mathrm{D}$ structure can be reconstructed from such an image series. Alternatively, single-particle $3 \mathrm{D}$ reconstruction was recently used to identify 5-10 $\mathrm{nm}$ silica structures with a dodecahedral shape. Here, a machine-learning algorithm was used to sort 10000 TEM images, taken randomly on structures with different orientations. ${ }^{8}$

Recently, atomically precise structures of Pt nanocrystals obtained through liquid cell TEM imaging were published, see Figure $3 .^{52}$ The apparently monodisperse ensemble still contained nanocrystals featuring differences in interatomic distances, internal defects, and strain. Moreover, the nanocrystal core appeared more ordered, and the surface more disordered. It is a telling result that exemplifies the limits to atomic precision discussed before. Hence, upon accepting the reality of heterogeneous ensembles in nanocrystal research, a key question becomes whether atomically precise average structures can represent nanocrystal ensembles, and how to define and determine these. Studies focusing on such average structures will need high throughput single nanocrystal analysis or - often the more straightforward approach - ensemble measurements.

\section{ATOMICALLY PRECISE REPRESENTATIONS}

Atomically precise structures have been used for years to represent nanocrystals in an ensemble and relate properties to structure. Electronic structure calculations by atomistic methods, such as tight-binding (TB), pseudopotential (PP), or relaxed structure determination by density functional theory (DFT) are impossible without explicit structure models.59-62 In the case of binary compounds such as PbSe, nanocrystal models were used to interpret the increasing the $\mathrm{Pb}: \mathrm{Se}$ ratio with decreasing size as a $\mathrm{Pb}$ surface excess. ${ }^{63}$ More recently, a realistic surface termination of nanocrystals was included to investigate the occurrence of electronic surface states, 7 the ligand binding energy, ${ }^{41}$ or the interaction between ligands and the surrounding solvent. ${ }^{64}$ Given this ongoing evolution, the question is whether and how ensembles can be represented by a single, atomically precise average nanocrystal. This is by no means an obvious point. What average structure should, for example, represent an ensemble consisting of an equal number of cubes and tetrahedrons? In addition, relying on atomically precise averages only make sense when impurity-related batch-tobatch variations in synthesis outcome are minimized. ${ }^{65-66}$ However, in case an average structure makes sense, the actual question is how the characteristics of such an average, atomically precise nanocrystal can be determined.

Probably the most unequivocal property when talking about ensemble averaging is the nanocrystal mass. Using Matrix-Assisted Laser Desorption/Ionization, coupled with Time of Flight Mass Spectrometry (MALDI-TOF MS), researchers determined the mass of Au clusters, ${ }^{67}$ metal oxide nanocrystals, ${ }^{68}$ and semiconductor nanocrystals. ${ }^{69}$ MALDI is a soft ionization technique, that can avoid fragmentation of the nanocrystal core and leave ligands attached. For atomically precise ensembles, this allows measuring the mass of the total structure with high resolution, fully consistent with the theoretical isotope distribution. As demonstrated for the $\mathrm{Au}_{25}\left(\mathrm{SCH}_{2} \mathrm{CH}_{2} \mathrm{Ph}\right)_{18}$ cluster, ${ }^{67}$ this approach is atomically precise and provides mass and composition in a single chemical formula. However, when lacking atomically precise ensembles, MS has not been applied to determine the chemical formula of nanocrystals. The inherent polydispersity of the NCs causes excessive peak overlap. Instead of a single peak, a broad distribution of masses is obtained. Still, while information on composition is lost, such a distribution does yield the average nanocrystal mass. Alternative methods to measure average masses - or rather the nanocrystal volume - involve direct imaging through TEM or small-angle $\mathrm{x}$-ray scattering (SAXS). While both techniques have limitations - mostly because they do not give a direct measurement of the nanocrystal mass consistent analysis results have been demonstrated for MALDI, TEM and SAXS, especially for nanocrystals larger than $\sim 5$ nm. .68

An average mass does not entail an average structure. While advanced TEM-based diffraction and imaging can yield atomically precise structures on specific nanocrystals, ensemble measurements are often more widely applicable. Sample damage is generally less of an 
issue and the parallel rather than serial analysis of multiple nanocrystals makes for more time-efficient ensemble averaging. To determine crystal structures, powder diffraction is a first method of choice..$^{70}$ However, crystals below $10 \mathrm{~nm}$ suffer from considerable peak broadening,

precluding structure determination of nanocrystals with atomic precision..$^{71}$ Analysis of total scattering by synchrotron X-rays or neutrons works around this problem. In total scattering, both the Bragg and diffuse scattering are measured up to large scattering vectors..$^{72}$ From the recorded scattering pattern, the pair distribution function (PDF) is calculated, which yields the distribution of distances between atom pairs in the nanocrystal.

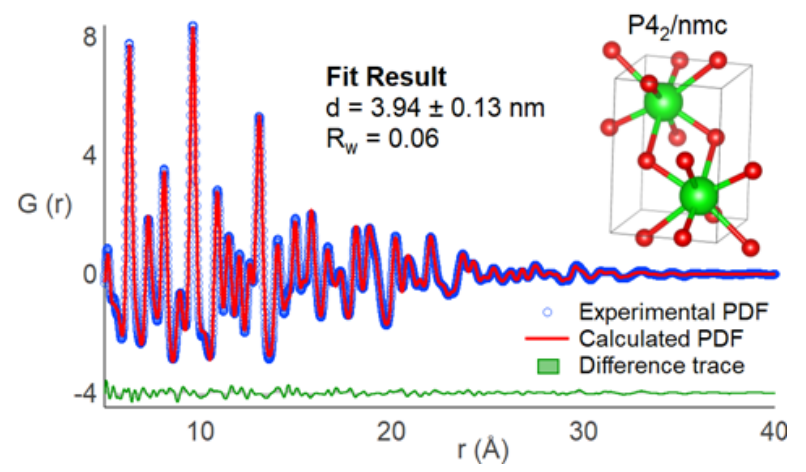

Figure 4. Attenuated crystal PDF modeling of monodisperse zirconia nanocrystals with a diameter of $4 \mathrm{~nm}$. See ref $73-74$ for details.

Experimental PDFs are regularly analyzed by two approaches. Attenuated-crystal models are built from a unit cell, expanded in three dimensions, and the finite nanocrystal size is accounted for by a spherical envelope function. ${ }^{75}$ As shown in Figure 4, the experimental PDF recorded on an ensemble of monodisperse, high-quality $\mathrm{ZrO}_{2}$ nanocrystals could be modeled in this way with residuals close to bulk standards. ${ }^{74}$ Alternatively, atomically precise nanocrystal structures can be built atom-by-atom and evaluated through discrete modeling. By not using an envelope function to terminate the nanocrystal, a more accurate, atomically precise structure model can be obtained. This approach is especially relevant in the case of complex nanostructures, such as decahedrally twinned metal nanocrystals, 75 or tetrahedral CdSe clusters. ${ }^{76-77}$ By fitting entire libraries of nanocrystal models with different structural motifs and sizes, an optimal average structure can be identified, ${ }^{78}$ but absolute atomic precision remains difficult to achieve due to degenerate solutions with a similar goodness-of-fit. A drawback of $x$-ray PDF analysis is the emphasis on the heavy atoms of the core, since light elements in the ligands have a much lower scattering cross-section. In contrast, neutron PDF can also integrate ligands in the modeling, 79 but reports on the precise analysis of nanocrystal surfaces by neutron scattering are still lacking.

Routine techniques to identify surface ligands are ${ }^{1} \mathrm{H}$ and ${ }^{31} \mathrm{P}$ solution and solid-state Nuclear Magnetic Resonance (NMR) spectroscopy.9, 73, 80-85 However, when used to study ligand-nanocrystal coordination, these techniques do not yield the atomically precise structure information they are renowned for. Take the example of the
$\operatorname{In}_{37} \mathrm{P}_{20}\left(\mathrm{O}_{2} \mathrm{CCH}_{2} \mathrm{Ph}\right)_{5^{1}}$ cluster, the structure of which was determined by single crystal XRD. Four different binding modes (symmetric bridging, asymmetric bridging, bidentate and bidentate+dative) were identified for the phenylacetate ligands, leading to 20 different coordination environments for 51 ligands!25 This points towards a particular impediment when analyzing nanocrystal ligands through NMR spectroscopy: even in an atomically precise ensemble, ligands will occupy hundreds of chemically different binding sites. A study on ligand desorption from CdSe nanocrystals showed the tip of this heterogeneity iceberg. By combining NMR spectroscopy and DFT simulations, it was found that CdSe nanocrystal surfaces contain a multitude of nanocrystal-ligand interactions with varying binding affinity. ${ }^{41}$ For structural analysis, this is a deadlock. To directly distinguish different binding sites, each site should lead to ligand resonances with distinct chemical shifts. The most likely end result, however, is a multitude of small chemical shifts leading to a single, heterogeneously broadened resonance in which atomically precise structural information is lost., unless specific binding modes dominate ${ }^{86}$ Most likely, any spectroscopic technique will face this issue when analyzing nanocrystal-ligand binding. Even solid-state NMR struggles to go beyond a mere distinction between surface atoms and core atoms; a far cry from an atomically precise picture of surface structures. ${ }^{87-88}$

Despite the limitations for structural characterization of nanocrystal surfaces, NMR spectroscopy can provide unique chemical insight in the ligand-surface interaction. $^{82}$ This information is most conveniently summarized through the ligand binding motif, where Green's ligand classification has been introduced to identify different ligand-nanocrystal bonds in the case of metal, metal oxide and semiconductor nanocrystals. ${ }^{89}$ Binding motifs are now used to describe nanocrystals through semi-quantitative chemical formulae. $9^{\circ}$ These distinguish between core and surface composition, and enable nanocrystals to be treated as compounds in chemical reactions. 


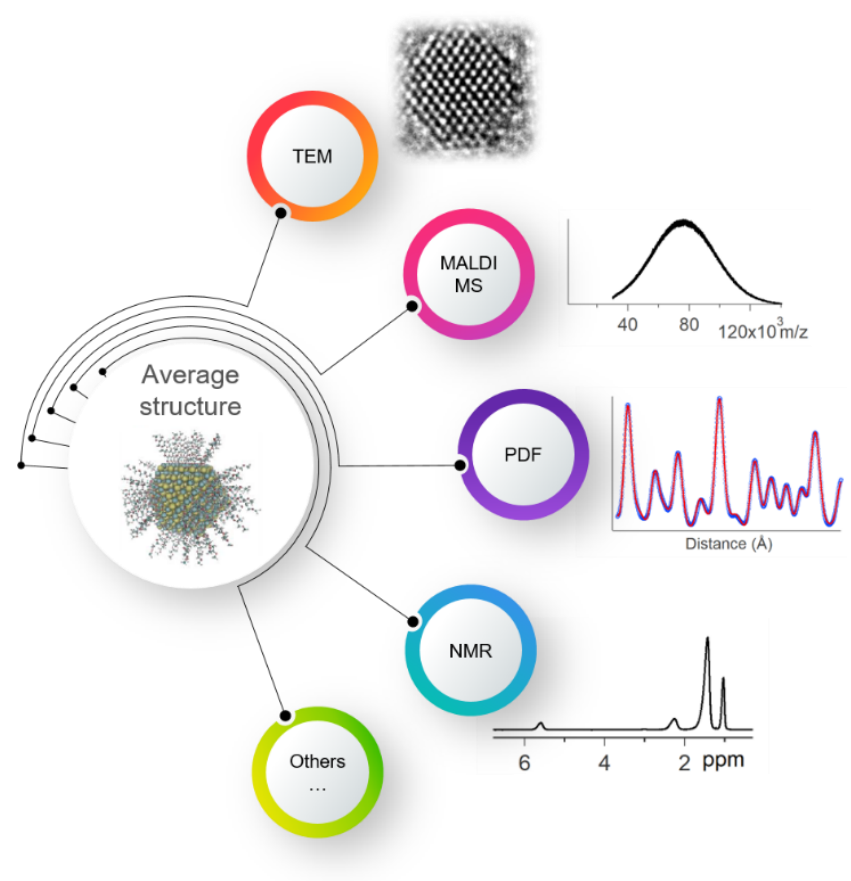

Figure 5. Average nanocrystal structures that represent a nanocrystal ensemble can be established through a combination of experimental methods that address composition, structure and surface chemistry.

What the above overview makes clear, is that no single technique will give an atomically precise, average structure that represents a nanocrystal ensemble. On the other hand, as highlighted in Figure 5, the combination of several techniques, like the ones discussed above and others, provides a clear pathway to obtain such average structures. For polydisperse ensembles, the average chemical formula can be obtained by analyzing mass or volume through MALDI, SAXS or TEM and composition and the ligand fraction through Rutherford Back Scattering or thermogravimetry, and quantitative NMR, respectively. ${ }^{85}$, 91 SAXS data (shape and size) can be linked with PDF (crystal structure) to derive a more precise atomic model of the nanocrystal core.92-93 NMR spectroscopy can connect both elements through the ligand binding motif. Ideally, structure solving would be performed in a global optimization algorithm that takes into account the boundary conditions provided by the data of many different characterization techniques. ${ }^{71}$

Atomically precise averages are increasingly used to study nanocrystals through a combination of experimental research and modeling at different length scales.7, 64, 94-100 In the case of semiconductor nanocrystals, the use of structural models with experimentally verified ligandnanocrystal interactions greatly enhanced the scope of electronic structure calculations. While initial studies using tight-binding or pseudopotential theory focused on the nanocrystal core - thereby terminating the surface by mathematical constructs such as pseudo-hydrogen ${ }^{101}$ or ligand potentials ${ }^{60}$ - DFT methods could shift from using hypothetical structures ${ }^{62}$ to comprehensive nanocrystal models that reflect the nanocrystal composition and the nanocrystal-ligand binding motif.7, 94 In this way, the relation between surface termination and the formation of surface-localized electronic states was laid bare, and successful strategies to passivate nanocrystal surfaces were discovered. ${ }^{102}$ Moreover, this approach not only enhanced understanding of structure-property relations in nanocrystals, but also widened the research scope from the properties of the nanocrystal core to the binding and packing of ligands ${ }^{100}$ and the interaction of nanocrystals with their surroundings. ${ }^{64}$ Hence the importance of constructing atomically precise average nanocrystal structures.

Despite the potential of atomically precise average structures to advance nanocrystal research, no clear rules have been formulated as to how the best structure should be identified. Moreover, while a single average structure may well represent a highly monodisperse ensemble, a proper description of polydisperse ensembles may need a set of different structures. Finally, while such issues can be sorted out in the near future, the fact remains that highly involved analysis will not suffice to amplify specific structure-property relations; this will require the formation of atomically precise nanocrystals.

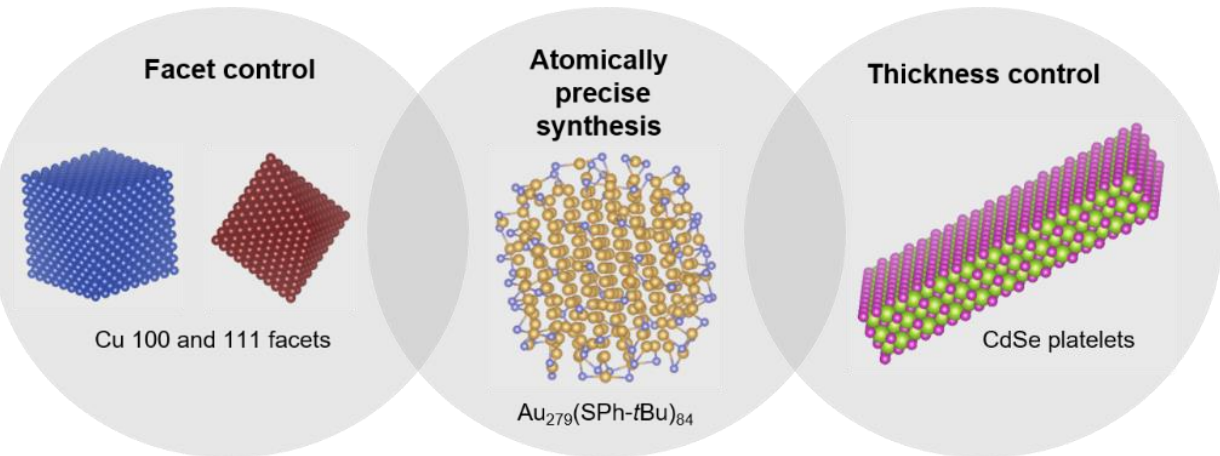

Figure 6. While Au nanocrystals synthesis has achieved the formation of $2.2 \mathrm{~nm}$ Au with atomic precison, other fields have mastered atomic precision light where only one aspect (thickness or exposed facets) is controlled with atomic precision.

\section{SYNTHESIS WITH ATOMIC PRECISION}

Most likely, the largest atomically precise nanocrystals formed are $2.2 \mathrm{~nm} \mathrm{Au}_{279}$ clusters. For other materials, such as $\mathrm{CdSe}$, InP and $\mathrm{ZrO}_{2}$, atomic precision is only achieved at much smaller sizes. ${ }^{25}, 76,103$ Of course, a perfectly monodisperse material can only be formed if structures exist that correspond to a sufficiently deep free energy 
minimum, i.e., magic sizes. In such a case, adding or subtracting an atom is highly unfavorable, and only the desired structure is formed. This principle is reflected in the gold cluster series with tert-butylbenzene thiol

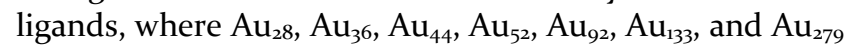
form but not the intermediate structures. 53 Several approaches lead to pure Au clusters. ${ }^{104}$ By fractionation, a mixture of clusters is separated in atomically precise sub ensembles. Upon size focusing, the mixture is exposed to severe conditions, under which only the stable clusters persist and unstable clusters are dissolved. During etching, larger nanocrystals are partially dissolved until a stable cluster is formed. In the transformation strategy, one stable cluster is converted in another by exposing the original cluster to different ligands. Finally, using kinetic control, the reduction reaction is slowed down and such a controlled reaction favors a single cluster size. It appears that stable cluster sizes are mostly determined by geometric considerations and ligand binding. ${ }^{53},{ }^{104}$ In the case of semiconductor or ceramic materials, atomically precise clusters are typically synthesized from highly reactive precursors at low to moderate temperatures $(-78$ $\left.-100{ }^{\circ} \mathrm{C}\right) .{ }^{25}, 76,105$ This stands in contrast to the synthesis of their nanocrystal counterpart, which is typically performed at significantly higher temperatures $(240-340$ $\left.{ }^{\circ} \mathrm{C}\right)$. Undoubtedly, thermal energy should be limited in cluster synthesis since it allows to overcome activation barriers, away from the intended atomically precise structure.

It thus appears that the synthesis of nanocrystals (>2 nm) with atomic precision in all its aspects remains an outstanding challenge. It is useful to draw the parallel with organic chemistry, where often even single-step reactions feature byproducts, and purification of the target compounds is necessary. As a consequence, multistep reaction sequences often take a dramatic hit in overall yield, even when executed by a highly skilled chemist. Taking into account that nanocrystal nucleation and growth involves thousands of reactions, it is almost surprising that relatively monodisperse nanocrystals can be formed in high yield, albeit not with atomic precision. Efforts into focusing the nanocrystal ensemble during crystal growth, ${ }^{10}$ are helpful to some extent but cannot achieve atomic precision. They are a poor man's implementation of an ideal. The question presents itself whether striving for atomic precision is worth the effort since for many applications, the properties of a structure with a few atoms more or less is not significantly affected. In this case, we can set more realistic goals and aim for a light version of atomic precision, which can well suffice to amplify specific nanocrystal properties.

A particular example of atomic precision light are colloidal nanoplatelets. ${ }^{106}$ These are flat sheets of semiconductor that are atomically precise in one dimension (thickness) while their width and length are polydisperse. The atomic precision in one dimension leads to exceptionally narrow photoluminescence lines (fwhm $<15 \mathrm{~nm})$. Since a narrow fwhm is important for optical applications, nanoplatelets represent a new paradigm. Spherical nanocrystals with narrow size dispersions on their diameter are suddenly outcompeted by objects with atomic precision in a single dimension. Such nanoplatelets are typically synthesized by a combination of short and long-chain carboxylate ligands. Once the symmetry is broken, the preference for lateral growth is explained by the smaller nucleus size on the edge of a platelet compared to a large facet. ${ }^{107}$ Even ripening usually a contributor to increased polydispersity proceeds in discrete phases, where two-monolayer platelets are sequentially converted to three-monolayer and then to four-monolayer platelets. ${ }^{108}$ These discrete transitions are accompanied by discrete shifts in the UVVis and photoluminescence spectrum. ${ }^{109}$ Fine tuning of the emission positon is thus limited; an intrinsic disadvantage of these systems. Interestingly, such atomic-precisionlight structures are unique model systems to investigate structure-property relations. In the case of CdSe nanoplatelets, a clear distinction could be made between strong ligand binding on facets and weak ligand binding on near-edge sites. ${ }^{39}$ Further passivating these latter states then leads to robust photoluminescence. ${ }^{110}$

Another example of atomic precision light is facet control. In heterogeneous catalysis, distinct facets often have a different activity or selectivity. ${ }^{111}$ For example, the (101) facets of anatase $\mathrm{TiO}_{2}$ appeared more active that the (oo1) facets in the catalytic photoreforming of methanol. ${ }^{19}$ In the reduction reaction of $\mathrm{CO}_{2}, \mathrm{Cu}$ (100) facets are more selective for ethylene, while $\mathrm{Cu}$ (111) facets are more selective toward methane. ${ }^{112-113}$ Small nanocrystals have an increased surface-to-volume ratio, which raises the number of catalytically active sites. While size-activity relations are observed in catalysis, perfect atomic precision is less important than exposing the right facets and edges.114-115 Facet control is typically achieved by preferential coordination of ligands on certain facets. For example, fluoride ions expose the (oo1) facet of anatase $\mathrm{TiO}_{2}{ }^{19}$ As a general rule, fast growing facets eliminate themselves. This means that fluoride ions bind strongly and selectively to the (oor) facet, thus preventing further growth. In fact, halides are versatile tools to tune the nanocrystal shape of many materials. ${ }^{116}$ Surfactants are another major tool to steer anisotropic growth. $\mathrm{TiO}_{2}$ nanorods were obtained by the addition of lauric acid to the reaction of titanium isopropoxide and titanium chloride in trioctylphosphine oxide. By using alkylphosphonic acids of different lengths and a seed mediated growth strategy, $\mathrm{CdSe} / \mathrm{CdS}$ nanorods with high aspect ratios were obtained. Furthermore, $\mathrm{Cu}$ nanocrystal spheres are obtained from $\mathrm{CuBr}$ in the presence of trioctylphosphine while $\mathrm{Cu}$ cubes are obtained in the presence of trioctylphosphine oxide, again showing the influence of the ligand. ${ }^{117}$

\section{CONCLUSION}

Starting from the idea that nanocrystals form a bridge between the world of small molecules and bulk materials, we explored the prospects of atomic precision in nanocrystal science. Based on a broad overview of materials and properties, we conclude that the propensity of nanocrystals to form defects may be a major impediment to atomic precision. On the other hand, several examples in the literature indicate that atomic precision light, where specific features of nanocrystals are 
controlled with atomic precision, is a realistic option that may well serve the needs of nanocrystal applications. On the other hand, it appears that major steps are being taken to achieve atomically precise analysis. Especially the combination of elemental, structural and chemical information opens a path to determine atomically precise average structures. Under some conditions, such averages can represent polydisperse ensembles and be used to explore structure/property relations through combined experimental/computational efforts. We believe this approach is readily applicable to a broad range of nanocrystals, and will have a profound impact on nanocrystal research in the years to come.

\section{AUTHOR INFORMATION}

\section{Corresponding Author}

zeger.hens@ugent.be

jonathan.deroo@unibas.ch

\section{ORCID}

Jonathan De Roo: oooo-0oo2-1264-9312

Zeger Hens: oooo-0oo2-7041-3375

\section{Notes}

The authors declare no competing financial interest

\section{ACKNOWLEDGMENTS}

Z.H. acknowledges support by FWO-Vlaanderen (research project 17006602) and Ghent University (BOF-GOA 01Go1019)

\section{REFERENCES}

1. Marty, J. J.; Oppenheim, R. C.; Speiser, P., Nanoparticles - New Colloidal Drug Delivery System. Pharm. Acta Helv. 1978, 53, 17-23.

2. Birringer, R.; Gleiter, H.; Klein, H. P.; Marquardt, P., Nanocrystalline Materials, an Approach to a Novel Solid Structure with Gas-Like Disorder. Phys. Lett. A 1984, 102, 365-369. 3. Kovalenko, M. V.; Manna, L.; Cabot, A.; Hens, Z.; Talapin, D. V.; Kagan, C. R.; Klimov, V. I.; Rogach, A. L.; Reiss, P.; Milliron, D. J.; Guyot-Sionnnest, P.; Konstantatos, G.; Parak, W. J.; Hyeon, T.; Korgel, B. A.; Murray, C. B.; Heiss, W., Prospects of Nanoscience with Nanocrystals. Acs Nano 2015, 9, 1012-1057.

4. $\quad$ Alivisatos, A. P., Perspectives on the Physical Chemistry of Semiconductor Nanocrystals. J. Phys. Chem. 1996, 100, 1322613239 .

5. Brus, L. E., A Simple-Model for the Ionization-Potential, Electron-Affinity, and Aqueous Redox Potentials of Small Semiconductor Crystallites. J. Chem. Phys. 1983, 79, 5566-5571.

6. Maes, J.; Castro, N.; De Nolf, K.; Walravens, W.; Abécassis, B.; Hens, Z., Size and Concentration Determination of Colloidal Nanocrystals by Small-Angle X-ray Scattering. Chem. Mat. 2018, 30, 3952-3962.

7. Houtepen, A. J.; Hens, Z.; Owen, J. S.; Infante, I., On the Origin of Surface Traps in Colloidal II-VI Semiconductor Nanocrystals. Chem. Mat. 2017, 29, 752-761.

8. Zhou, K. B.; Li, Y. D., Catalysis Based on Nanocrystals with Well-Defined Facets. Angew. Chem. Int. Ed. 2012, 51, 602-613.

9. De Roo, J.; Van Driessche, I.; Martins, J. C.; Hens, Z., Colloidal metal oxide nanocrystal catalysis by sustained chemically driven ligand displacement. Nat. Mater. 2016, 15, 517521.

10. Yin, Y.; Alivisatos, A. P., Colloidal Nanocrystal Synthesis and the Organic-Inorganic Interface. Nature 2oo5, 437, 664-670.
11. Peng, X. G.; Manna, L.; Yang, W. D.; Wickham, J.; Scher, E.; Kadavanich, A.; Alivisatos, A. P., Shape Control of CdSe Nanocrystals. Nature 2000, 404, 59-61.

12. Jun, Y.-w.; Casula, M. F.; Sim, J.-H.; Kim, S. Y.; Cheon, J.; Alivisatos, A. P., Surfactant-Assisted Elimination of a High Energy Facet as a Means of Controlling the Shapes of TiO2 Nanocrystals. J. Am. Chem. Soc. 2003, 125, 15981-15985.

13. Nikoobakht, B.; El-Sayed, M. A., Preparation and Growth Mechanism of Gold Nanorods (NRs) Using SeedMediated Growth Method. Chem. Mat. 2003, 15, 1957-1962.

14. Singh, S.; Liu, P.; Singh, A.; Coughlan, C.; Wang, J. J.; Lusi, M.; Ryan, K. M., Colloidal CuzZnSn(SSe)(4) (CZTSSe) Nanocrystals: Shape and Crystal Phase Control to Form Dots, Arrows, Ellipsoids, and Rods. Chem. Mat. 2015, 27, 4742-4748.

15. Ithurria, S.; Dubertret, B., Quasi 2D Colloidal CdSe Platelets with Thicknesses Controlled at the Atomic Level. J. Am. Chem. Soc. 2008, 130, 16504-16505.

16. Ithurria, S.; Tessier, M. D.; Mahler, B.; Lobo, R. P. S. M.; Dubertret, B.; Efros, A. L., Colloidal Nanoplatelets with TwoDimensional Electronic Structure. Nat. Mater. 2o11, 10, 936-941.

17. Akkerman, Q. A.; Motti, S. G.; Kandada, A. R. S.; Mosconi, E.; D'Innocenzo, V.; Bertoni, G.; Marras, S.; Kamino, B. A.; Miranda, L.; De Angelis, F.; Petrozza, A.; Prato, M.; Manna, L., Solution Synthesis Approach to Colloidal Cesium Lead Halide Perovskite Nanoplatelets with Monolayer-Level Thickness Control. J. Am. Chem. Soc. 2016, 138, 1010-1016.

18. Weidman, M. C.; Goodman, A. J.; Tisdale, W. A., Colloidal Halide Perovskite Nanoplatelets: An Exciting New Class of Semiconductor Nanomaterials. Chem. Mat. 2017, 29, 50195030 .

19. Gordon, T. R.; Cargnello, M.; Paik, T.; Mangolini, F.; Weber, R. T.; Fornasiero, P.; Murray, C. B., Nonaqueous Synthesis of $\mathrm{TiO}_{2}$ Nanocrystals Using $\mathrm{TiF}_{4}$ to Engineer Morphology, Oxygen Vacancy Concentration, and Photocatalytic Activity. J. Am. Chem. Soc. 2012, 134, 6751-6761.

2o. Schliehe, C.; Juarez, B. H.; Pelletier, M.; Jander, S.; Greshnykh, D.; Nagel, M.; Meyer, A.; Foerster, S.; Kornowski, A.; Klinke, C.; Weller, H., Ultrathin PbS Sheets by Two-Dimensional Oriented Attachment. Science 2010, 329, 550-553.

21. Joo, J.; Son, J. S.; Kwon, S. G.; Yu, J. H.; Hyeon, T., Lowtemperature solution-phase synthesis of quantum well structured CdSe nanoribbons. J. Am. Chem. Soc. 20o6, 128, 5632-5633.

22. Manna, L.; Scher, E. C.; Alivisatos, A. P., Synthesis of Soluble and Processable Rod-, Arrow-, Teardrop-, and TetrapodShaped CdSe Nanocrystals. J. Am. Chem. Soc. 20oo, 122, 1270012706.

23. Wang, J. J.; Liu, P.; Seaton, C. C.; Ryan, K. M., Complete Colloidal Synthesis of $\mathrm{Cu}_{2} \mathrm{SnSe} 3$ Nanocrystals with Crystal Phase and Shape Control. J. Am. Chem. Soc. 2o14, 136, 7954-796o.

24. Schmid, G., The Relevance of Shape and Size of Au-55 Clusters. Chem. Soc. Rev. 2oo8, 37, 1909-1930.

25. Gary, D. C.; Flowers, S. E.; Kaminsky, W.; Petrone, A.; Li, X.; Cossairt, B. M., Single-Crystal and Electronic Structure of a $1.3 \mathrm{~nm}$ Indium Phosphide Nanocluster. J. Am. Chem. Soc. 2016, $138,1510-1513$.

26. Sakthivel, N. A.; Theivendran, S.; Ganeshraj, V.; Oliver, A. G.; Dass, A., Crystal Structure of Faradaurate-279: Au279(SPhtBu) 84 Plasmonic Nanocrystal Molecules. J. Am. Chem. Soc. 2017, 139, 15450-15459.

27. Turnbull, D., Formation of Crystal Nuclei in Liquid Metals. J. Appl. Phys. 1950, 21, 1022-1028.

28. Dalpian, G. M.; Chelikowsky, J. R., Self-Purification in Semiconductor Nanocrystals. Phys. Rev. Lett. 20o6, 96, 226802.

29. Gao, P.; Ma, H.; Wu, Q.; Qiao, L.; Volinsky, A. A.; Su, Y., Size-Dependent Vacancy Concentration in Nickel, Copper, Gold, and Platinum Nanoparticles. J. Phys. Chem. C 2016, 120, 1761317619 . 
30. Li, Y.; Zang, L.; Jacobs, D. L.; Zhao, J.; Yue, X.; Wang, C., In Situ Study on Atomic Mechanism of Melting and Freezing of Single Bismuth Nanoparticles. Nat. Comm. 2017, 8, 14462.

31. Schiotz, J.; Di Tolla, F. D.; Jacobsen, K. W., Softening of Nanocrystalline Metals at Very Small Grain Sizes. Nature 1998, 391, 561-563.

32. Schiøtz, J.; Jacobsen, K. W., A Maximum in the Strength of Nanocrystalline Copper. Science 2003, 301, 1357.

33. Chen, B.; Lutker, K.; Raju, S. V.; Yan, J.; Kanitpanyacharoen, W.; Lei, J.; Yang, S.; Wenk, H.-R.; Mao, H.-k.; Williams, Q., Texture of Nanocrystalline Nickel: Probing the Lower Size Limit of Dislocation Activity. Science 2012, 338, 1448.

34. Parakh, A.; Lee, S.; Harkins, K. A.; Kiani, M. T.; Doan, D.; Kunz, M.; Doran, A.; Hanson, L. A.; Ryu, S.; Gu, X. W., Nucleation of Dislocations in $3.9 \mathrm{~nm}$ Nanocrystals at High Pressure. Phys. Rev. Lett. 2020, 124, 106104.

35. Hughes, S. M.; Alivisatos, A. P., Anisotropic Formation and Distribution of Stacking Faults in II-VI Semiconductor Nanorods. Nano Lett. 2013, 13, 106-110.

36. Johnson, C. L.; Snoeck, E.; Ezcurdia, M.; RodríguezGonzález, B.; Pastoriza-Santos, I.; Liz-Marzán, L. M.; Hÿtch, M. J., Effects of Elastic Anisotropy on Strain Distributions in Decahedral Gold Nanoparticles. Nat. Mater. 2oo8, 7, 120-124.

37. Garzon, I. L.; Michaelian, K.; Beltran, M. R.; PosadaAmarillas, A.; Ordejon, P.; Artacho, E.; Sanchez-Portal, D.; Soler, J. M., Lowest Energy Structures of Gold Nanoclusters. Phys. Rev. Lett. 1998, 81, 1600-1603.

38. Boles, M. A.; Ling, D.; Hyeon, T.; Talapin, D. V., The Surface Science of Nanocrystals. Nat. Mater. 2016, 15, 141.

39. Singh, S.; Tomar, R.; ten Brinck, S.; De Roo, J.; Geiregat, P.; Martins, J. C.; Infante, I.; Hens, Z., Colloidal CdSe Nanoplatelets, A Model for Surface Chemistry/Optoelectronic Property Relations in Semiconductor Nanocrystals. J. Am. Chem. Soc. 2018, 140, 13292-13300.

40. Anderson, N. C.; Hendricks, M. P.; Choi, J. J.; Owen, J. S., Ligand Exchange and the Stoichiometry of Metal Chalcogenide Nanocrystals: Spectroscopic Observation of Facile MetalCarboxylate Displacement and Binding. J. Am. Chem. Soc. 2013, 135, 18536-18548.

41. Drijvers, E.; De Roo, J.; Martins, J. C.; Infante, I.; Hens, Z., Ligand Displacement Exposes Binding Site Heterogeneity on CdSe Nanocrystal Surfaces. Chem Mater 2018, 30, 1178-1186.

42. Jain, P. K.; Beberwyck, B. J.; Fong, L. K.; Polking, M. J.; Alivisatos, A. P., Highly Luminescent Nanocrystals From Removal of Impurity Atoms Residual From Ion-Exchange Synthesis. Angew. Chem. Int. Ed. 2012, 51, 2387-2390.

43. Marri, I.; Degoli, E.; Ossicini, S., Doped and Codoped Silicon Nanocrystals: The Role of Surfaces and Interfaces. Prog. Surf. Sci. 2017, 92, 375-408.

44. Erwin, S. C.; Zu, L. J.; Haftel, M. I.; Efros, A. L.; Kennedy, T. A.; Norris, D. J., Doping Semiconductor Nanocrystals. Nature 2005, 436, 91-94.

45. Liu, M. Z.; Guyot-Sionnest, P., Mechanism of Silver(I)Assisted Growth of Gold Nanorods and Bipyramids. J. Phys. Chem. B 2005, 109, 22192-22200.

46. Sanchez-Iglesias, A.; Pastoriza-Santos, I.; Perez-Juste, J.; Rodriguez-Gonzalez, B.; de Abajo, F. J. G.; Liz-Marzan, L. M., Synthesis and Optical Properties of Gold Nanodecahedra with Size Control. Adv. Mater. 20o6, 18, 2529-+.

47. Carbone, L.; Nobile, C.; De Giorgi, M.; Sala, F. D.; Morello, G.; Pompa, P.; Hytch, M.; Snoeck, E.; Fiore, A.; Franchini, I. R.; Nadasan, M.; Silvestre, A. F.; Chiodo, L.; Kudera, S.; Cingolani, R.; Krahne, R.; Manna, L., Synthesis and MicrometerScale Assembly of Colloidal CdSe/CdS Nanorods Prepared by a Seeded Growth Approach. Nano Lett. 2007, 7, 2942-2950

48. Talapin, D. V.; Nelson, J. H.; Shevchenko, E. V.; Aloni, S.; Sadtler, B.; Alivisatos, A. P., Seeded Growth of Highly Luminescent CdSe/CdS Nanoheterostructures with Rod and Tetrapod Morphologies. Nano Lett. 2007, 7, 2951-2959.
49. Billet, J.; Dujardin, W.; De Keukeleere, K.; De Buysser, K.; De Roo, J.; Van Driessche, I., Size Tunable Synthesis and Surface Chemistry of Metastable TiO2-Bronze Nanocrystals. Chem. Mat. 2018, 30, 4298-4306.

50. Coughlan, C.; Ibanez, M.; Dobrozhan, O.; Singh, A.; Cabot, A.; Ryan, K. M., Compound Copper Chalcogenide Nanocrystals. Chem. Rev. 2017, 117, 5865-6109.

51. Hennig, C.; Weiss, S.; Kraus, W.; Kretzschmar, J.; Scheinost, A. C., Solution Species and Crystal Structure of $\mathrm{Zr}(\mathrm{IV})$ Acetate. Inorg. Chem. 2017, 56, 2473-2480.

52. Kim, B. H.; Heo, J.; Kim, S.; Reboul, C. F.; Chun, H.; Kang, D.; Bae, H.; Hyun, H.; Lim, J.; Lee, H.; Han, B.; Hyeon, T.; Alivisatos, A. P.; Ercius, P.; Elmlund, H.; Park, J., Critical differences in $3 \mathrm{D}$ atomic structure of individual ligand-protected nanocrystals in solution. Science 2020, 368, 60-67.

53. Sakthivel, N. A.; Dass, A., Aromatic Thiolate-Protected Series of Gold Nanomolecules and a Contrary Structural Trend in Size Evolution. Acc. Chem Res. 2018, 51, 1774-1783.

54. Shevchenko, E. V.; Talapin, D. V.; Kotov, N. A.; O'Brien, S.; Murray, C. B., Structural Diversity in Binary Nanoparticle Superlattices. Nature 2006, 439, 55-59.

55. Chapman, H. N., X-Ray Free-Electron Lasers for the Structure and Dynamics of Macromolecules. In Ann. Rev. Biochem., Vol 88, Kornberg, R. D., Ed. 2019; Vol. 88, pp 35-58.

56. Gemmi, M.; Mugnaioli, E.; Gorelik, T. E.; Kolb, U.; Palatinus, L.; Boullay, P.; Hovmoller, S.; Abrahams, J. P., 3D Electron Diffraction: The Nanocrystallography Revolution. ACS Cent. Sci. 2019, 5, 1315-1329.

57. Park, J.; Elmlund, H.; Ercius, P.; Yuk, J. M.; Limmer, D. T.; Chen, Q.; Kim, K.; Han, S. H.; Weitz, D. A.; Zettl, A.; Alivisatos, A. P., 3D Structure of Individual Nanocrystals in Solution by Electron Microscopy. Science 2015, 349, 290-295.

58. Ma, K.; Gong, Y. Y.; Aubert, T.; Turker, M. Z.; Kao, T.; Doerschuk, P. C.; Wiesner, U., Self-Assembly of Highly Symmetrical, Ultrasmall Inorganic Cages Directed by Surfactant Micelles. Nature 2018, 558, 577-+.

59. Lippens, P. E.; Lannoo, M., Calculation of the Band-Gap for Small CdS and ZnS Crystallites. Phys. Rev. B 1989, 39, 1093510942.

6o. Wang, L. W.; Zunger, A., Pseudopotential Calculations of Nanoscale CdSe Quantum Dots. Phys. Rev. B 1996, 53, 95799582 .

61. Niquet, Y. M.; Delerue, C.; Allan, G.; Lannoo, M., Method for Tight-Binding Parametrization: Application to Silicon Nanostructures. Phys. Rev. B 200o, 62, 5109-5116.

62. Puzder, A.; Williamson, A. J.; Zaitseva, N.; Galli, G.; Manna, L.; Alivisatos, A. P., The Effect of Organic Ligand Binding on the Growth of CdSe Nanoparticles Probed by Ab Initio Calculations. Nano Lett. 2oo4, 4, 2361-2365.

63. Moreels, I.; Lambert, K.; De Muynck, D.; Vanhaecke, F.; Poelman, D.; Martins, J. C.; Allan, G.; Hens, Z., Composition and Size-Dependent Extinction Coefficient of Colloidal PbSe Quantum Dots. Chem. Mat. 2007, 19, 6101-6106.

64. De Roo, J.; Yazdani, N.; Drijvers, E.; Lauria, A.; Maes, J.; Owen, J. S.; Van Driessche, I.; Niederberger, M.; Wood, V.; Martins, J. C.; Infante, I.; Hens, Z., Probing Solvent-Ligand Interactions in Colloidal Nanocrystals by the NMR Line Broadening. Chem. Mat. 2018, 30, 5485-5492.

65. Wang, F. D.; Tang, R.; Buhro, W. E., The Trouble with TOPO; Identification of Adventitious Impurities Beneficial to the Growth of Cadmium Selenide Quantum Dots, Rods, and Wires. Nano Lett. 2008, 8, 3521-3524.

66. Dhaene, E.; Billet, J.; Bennett, E.; Van Driessche, I.; De Roo, J., The Trouble with ODE: Polymerization during Nanocrystal Synthesis. Nano Lett. 2019, 19, 7411-7417.

67. Dass, A.; Stevenson, A.; Dubay, G. R.; Tracy, J. B.; Murray, R. W., Nanoparticle MALDI-TOF Mass Spectrometry without Fragmentation: $\mathrm{Au}_{2}\left(\mathrm{SCH}_{2} \mathrm{CH}_{2} \mathrm{Ph}\right)_{18} 8$ and Mixed 
Monolayer Au25 $\left(\mathrm{SCH}_{2} \mathrm{CH}_{2} \mathrm{Ph}\right)_{18-x}(\mathrm{~L}) x$. J. Am. Chem. Soc. 2oo8, 130, 5940-5946.

68. Kim, B. H.; Shin, K.; Kwon, S. G.; Jang, Y.; Lee, H.-S.; Lee, H.; Jun, S. W.; Lee, J.; Han, S. Y.; Yim, Y.-H.; Kim, D.-H.; Hyeon, T., Sizing by Weighing: Characterizing Sizes of Ultrasmall-Sized Iron Oxide Nanocrystals Using MALDI-TOF Mass Spectrometry. J. Am. Chem. Soc. 2013, 135, 2407-2410.

69. Xie, L.; Shen, Y.; Franke, D.; Sebastián, V.; Bawendi, M. G.; Jensen, K. F., Characterization of Indium Phosphide Quantum Dot Growth Intermediates Using MALDI-TOF Mass Spectrometry. J. Am. Chem. Soc. 2016, 138, 13469-13472.

7o. Holder, C. F.; Schaak, R. E., Tutorial on Powder X-ray Diffraction for Characterizing Nanoscale Materials. ACS Nano 2019, 13, 7359-7365.

71. Billinge, S. J. L.; Levin, I., The Problem with Determining Atomic Structure at the Nanoscale. Science 2007, 316, 561-565.

72. Billinge, S. J. L., Nanoscale Structural Order from the Atomic Pair Distribution Function (PDF): There's Plenty of Room in the Middle. J. Sol. Stat. Chem. 2oo8, 181, 1695-170o.

73. De Keukeleere, K.; Coucke, S.; De Canck, E.; Van Der Voort, P.; Delpech, F.; Coppel, Y.; Hens, Z.; Van Driessche, I.; Owen, J. S.; De Roo, J., Stabilization of Colloidal Ti, Zr, and Hf Oxide Nanocrystals by Protonated Tri-n-octylphosphine Oxide (TOPO) and Its Decomposition Products. Chem. Mat. 2017, 29, 10233-10242.

74. Rijckaert, H.; De Roo, J.; Van Zele, M.; Banerjee, S.; Huhtinen, H.; Paturi, P.; Bennewitz, J.; Billinge, S.; Bäcker, M.; De Buysser, K.; Van Driessche, I., Pair Distribution Function Analysis of $\mathrm{ZrO}_{2}$ Nanocrystals and Insights in the Formation of $\mathrm{ZrO}_{2}-$ $\mathrm{YBa}_{2} \mathrm{Cu}_{3} \mathrm{O}_{7}$ Nanocomposites. Mater. 2018, 11, 1066.

75. Banerjee, S.; Liu, C.-H.; Lee, J. D.; Kovyakh, A.; Grasmik, V.; Prymak, O.; Koenigsmann, C.; Liu, H.; Wang, L.; Abeykoon, A. M. M.; Wong, S. S.; Epple, M.; Murray, C. B.; Billinge, S. J. L., Improved Models for Metallic Nanoparticle Cores from Atomic Pair Distribution Function (PDF) Analysis. J. Phys. Chem. C 2018, 122, 29498-29506.

76. Beecher, A. N.; Yang, X.; Palmer, J. H.; LaGrassa, A. L.; Juhas, P.; Billinge, S. J. L.; Owen, J. S., Atomic Structures and Gram Scale Synthesis of Three Tetrahedral Quantum Dots. J. Am. Chem. Soc. 2014, 136, 10645-10653.

77. Jensen, K. M. O.; Juhas, P.; Tofanelli, M. A.; Heinecke, C. L.; Vaughan, G.; Ackerson, C. J.; Billinge, S. J. L., Polymorphism in Magic-Sized Au-144(SR)(6o) Clusters. Nat. Comm. 2016, 7 , 11859 .

78. Banerjee, S.; Liu, C.-H.; Jensen, K. M. O.; Juhas, P.; Lee, J. D.; Tofanelli, M.; Ackerson, C. J.; Murray, C. B.; Billinge, S. J. L., Cluster-Mining: an Approach for Determining Core Structures of Metallic Nanoparticles from Atomic Pair Distribution Function Data. Acta Cryst. A 2020, 76, 24-31.

79. $\quad$ Page, K.; Proffen, T.; Niederberger, M.; Seshadri, R., Probing Local Dipoles and Ligand Structure in $\mathrm{BaTiO}_{3}$ Nanoparticles. Chem. Mat. 2010, 22, 4386-4391.

8o. Moreels, I.; Fritzinger, B.; Martins, J. C.; Hens, Z., Surface Chemistry of Colloidal PbSe Nanocrystals. J. Am. Chem. Soc. 2008, 130, 15081-15086.

81. Owen, J. S.; Park, J.; Trudeau, P. E.; Alivisatos, A. P., Reaction Chemistry and Ligand Exchange at Cadmium-Selenide Nanocrystal Surfaces. J. Am. Chem. Soc. 20o8, 130, 12279-1228o.

82. Hens, Z.; Martins, J. C., A Solution NMR Toolbox for Characterizing the Surface Chemistry of Colloidal Nanocrystals. Chem. Mat. 2013, 25, 1211-1221.

83. Marbella, L. E.; Millstone, J. E., NMR Techniques for Noble Metal Nanoparticles. Chem. Mat. 2015, 27, 2721-2739.

84. Berestok, T.; Guardia, P.; Blanco, J.; Nafria, R.; Torruella, P.; Lopez-Conesa, L.; Estrade, S.; Ibanez, M.; de Roo, J.; Luo, Z.; Cadavid, D.; Martins, J. C.; Kovalenko, M. V.; Peiro, F.; Cabot, A., Tuning Branching in Ceria Nanocrystals. Chem. Mat. 2017, 29, 4418-4424.
85. Grigel, V.; Sagar, L. K.; De Nolf, K.; Zhao, Q.; Vantomme, A.; De Roo, J.; Infante, I.; Hens, Z., The Surface Chemistry of Colloidal HgSe Nanocrystals, Towards Stoichiometric Quantum Dots by Design. Chem. Mat. 2018, 30, 7637-7647

86. Zhang, J.; Zhang, H.; Cao, W.; Pang, Z.; Li, J.; Shu, Y.; Zhu, C.; Kong, X.; Wang, L.; Peng, X., Identification of FacetDependent Coordination Structures of Carboxylate Ligands on CdSe Nanocrystals. J. Am. Chem. Soc. 2019, 141, 15675-15683.

87. Piveteau, L.; Ong, T. C.; Rossini, A. J.; Emsley, L.; Coperet, C.; Kovalenko, M. V., Structure of Colloidal Quantum Dots from Dynamic Nuclear Polarization Surface Enhanced NMR Spectroscopy. J. Am. Chem. Soc. 2015, 137, 13964-71.

88. Tessier, M. D.; Baquero, E. A.; Dupont, D.; Grigel, V.; Bladt, E.; Bals, S.; Coppel, Y.; Hens, Z.; Nayral, C.; Delpech, F., Interfacial Oxidation and Photoluminescence of InP-Based Core/Shell Quantum Dots. Chem. Mat. 2018, 30, 6877-6883.

89. Anderson, N. C.; Hendricks, M. P.; Choi, J. J.; Owen, J. S., Ligand Exchange and the Stoichiometry of Metal Chalcogenide Nanocrystals: Spectroscopic Observation of Facile MetalCarboxylate Displacement and Binding. Journal of the American Chemical Society 2013, 135, 18536-18548.

9o. De Roo, J.; De Keukeleere, K.; Hens, Z.; Van Driessche, I., From ligands to binding motifs and beyond; the enhanced versatility of nanocrystal surfaces. Dalton. Trans. 2016, 45, 1327783.

91. Fritzinger, B.; Capek, R. K.; Lambert, K.; Martins, J. C.; Hens, Z., Utilizing Self-Exchange To Address the Binding of Carboxylic Acid Ligands to CdSe Quantum Dots. J. Am. Chem. Soc. 2010, 132, 10195-10201.

92. Hua, X.; Liu, Z.; Bruce, P. G.; Grey, C. P., The Morphology of $\mathrm{TiO}_{2}$ (B) Nanoparticles. J. Am. Chem. Soc. 2015, 137, 13612-23.

93. Moscheni, D.; Bertolotti, F.; Piveteau, L.; Protesescu, L.; Dirin, D. N.; Kovalenko, M. V.; Cervellino, A.; Pedersen, J. S.; Masciocchi, N.; Guagliardi, A., Size-Dependent Fault-Driven Relaxation and Faceting in Zincblende CdSe Colloidal Quantum Dots. ACS Nano 2018, 12, 12558-12570.

94. Zherebetskyy, D.; Scheele, M.; Zhang, Y. J.; Bronstein, N.; Thompson, C.; Britt, D.; Salmeron, M.; Alivisatos, P.; Wang, L. W., Hydroxylation of the surface of $\mathrm{PbS}$ nanocrystals passivated with oleic acid. Science 2014, 344, 1380-1384.

95. Bozyigit, D.; Yazdani, N.; Yarema, M.; Yarema, O.; Lin, W. M. M.; Volk, S.; Vuttivorakulchai, K.; Luisier, M.; Juranyi, F.; Wood, V., Soft surfaces of nanomaterials enable strong phonon interactions. Nature 2016, 531, 618-622.

96. Kirkwood, N.; Monchen, J. O. V.; Crisp, R. W.; Grimaldi, G.; Bergstein, H. A. C.; du Fosse, I.; van der Stam, W.; Infante, I.; Houtepen, A. J., Finding and Fixing Traps in II-VI and III-V Colloidal Quantum Dots: The Importance of Z-Type Ligand Passivation. J. Am. Chem. Soc. 2018, 140, 15712-15723.

97. Bodnarchuk, M. I.; Boehme, S. C.; ten Brinck, S.; Bernasconi, C.; Shynkarenko, Y.; Krieg, F.; Widmer, R.; Aeschlimann, B.; Gunther, D.; Kovalenko, M. V.; Infante, I., Rationalizing and Controlling the Surface Structure and Electronic Passivation of Cesium Lead Halide Nanocrystals. ACS Energy Lett. 2019, 4, 63-74.

98. Boehme, S. C.; Ten Brinck, S.; Maes, J.; Yazdani, N.; Zapata, F.; Chen, K.; Wood, V.; Hodgkiss, J. M.; Hens, Z.; Geiregat, P.; Infante, I., Phonon-Mediated and Weakly Size-Dependent Electron and Hole Cooling in $\mathrm{CsPbBr}_{3}$ Nanocrystals Revealed by Atomistic Simulations and Ultrafast Spectroscopy. Nano Lett. 2020, 20, 1819-1829.

99. Delerue, C.; Vanmaekelbergh, D., Electronic Band Structure of Zinc Blende CdSe and Rock Salt PbSe Semiconductors with Silicene-Type Honeycomb Geometry. $2 D$ Mater. 2015, 2, 13.

10o. De Nolf, K.; Cosseddu, S. M.; Jasieniak, J. J.; Drijvers, E.; Martins, J. C.; Infante, I.; Hens, Z., Binding and Packing in Two- 
Component Colloidal Quantum Dot Ligand Shells: Linear versus Branched Carboxylates. J. Am. Chem. Soc. 2017, 139, 3456-3464.

101. Allan, G.; Delerue, C.; Lannoo, M., Electronic Structure of Amorphous Silicon Nanoclusters. Phys. Rev. Lett. 1997, 78, 31613164 .

102. Kirkwood, N.; Monchen, J. O. V.; Crisp, R. W.; Grimaldi, G.; Bergstein, H. A.; du Fossé, I.; van der Stam, W.; Infante, I.; Houtepen, A. J., Finding and fixing traps in II-VI and III-V colloidal quantum dots: The importance of Z-type ligand passivation. J. Am. Chem. Soc. 2018, 140, 15712-15723.

103. Sun, Q.; Liu, C.; Zhang, G.; Zhang, J.; Tung, C.-H.; Wang, Y., Aqueous Isolation of $17-\mathrm{Nuclear} \mathrm{Zr} / \mathrm{Hf}$ Oxide Clusters during the Hydrothermal Synthesis of $\mathrm{ZrO}_{2} / \mathrm{HfO}_{2}$. Chem. Eur. J. 2018, 24 , 14701-14706.

104. Kurashige, W.; Niihori, Y.; Sharma, S.; Negishi, Y., Precise Synthesis, Functionalization and Application of ThiolateProtected Gold Clusters. Coord. Chem. Rev. 2016, 320-321, 238-25o.

105. Puchberger, M.; Kogler, F. R.; Jupa, M.; Gross, S.; Fric, H.; Kickelbick, G.; Schubert, U., Can the Clusters $\mathrm{Zr}_{6} \mathrm{O}_{4}(\mathrm{OH})(4)(\mathrm{OOCR})(12)$ and $\mathrm{Zr}_{4} \mathrm{O}_{4}(\mathrm{OH})(4)(\mathrm{OOCR})(12)(2)$ be Converted into Each Other? Eur. J. Inorg. Chem. 2oo6, 16, 32833293.

106. Nasilowski, M.; Mahler, B.; Lhuillier, E.; Ithurria, S.; Dubertret, B., Two-Dimensional Colloidal Nanocrystals. Chem. Rev. 2016, 116, 10934-10982.

107. Riedinger, A.; Ott, F. D.; Mule, A.; Mazzotti, S.; Knüsel, P. N.; Kress, Stephan J. P.; Prins, F.; Erwin, S. C.; Norris, D. J., An Intrinsic Growth Instability in Isotropic Materials Leads to QuasiTwo-Dimensional Nanoplatelets. Nat. Mater. 2017, 16, 743-748.

108. Ott, F. D.; Riedinger, A.; Ochsenbein, D. R.; Knüsel, P. N.; Erwin, S. C.; Mazzotti, M.; Norris, D. J., Ripening of Semiconductor Nanoplatelets. Nano Lett. 2017, 17, 6870-6877.

109. Christodoulou, S.; Climente, J. I.; Planelles, J.; Brescia, R.; Prato, M.; Martin-Garcia, B.; Khan, A. H.; Moreels, I., Chloride-
Induced Thickness Control in CdSe Nanoplatelets. Nano Lett. 2018, 18, 6248-6254.

110. Leemans, J.; Singh, S.; Li, C.; Ten Brinck, S.; Bals, S.; Infante, I.; Moreels, I.; Hens, Z., Near-Edge Ligand Stripping and Robust Radiative Exciton Recombination in $\mathrm{CdSe} / \mathrm{CdS}$ Core/Crown Nanoplatelets. J. Phys. Chem. Lett. 2020, 11, 33393344 .

111. Cargnello, M., Colloidal Nanocrystals as Building Blocks for Well-Defined Heterogeneous Catalysts. Chem. Mat. 2019, 31, 576-596.

112. Schouten, K. J. P.; Pérez Gallent, E.; Koper, M. T. M., Structure Sensitivity of the Electrochemical Reduction of Carbon Monoxide on Copper Single Crystals. ACS Catal. 2013, 3, 12921295 .

113. De Gregorio, G. L.; Burdyny, T.; Loiudice, A.; Iyengar, P.; Smith, W. A.; Buonsanti, R., Facet-Dependent Selectivity of Cu Catalysts in Electrochemical $\mathrm{CO}_{2}$ Reduction at Commercially Viable Current Densities. ACS Catal. 2020, 4854-4862.

114. Loiudice, A.; Lobaccaro, P.; Kamali, E. A.; Thao, T.; Huang, B. H.; Ager, J. W.; Buonsanti, R., Tailoring Copper Nanocrystals towards $\mathrm{C}_{2}$ Products in Electrochemical $\mathrm{CO}_{2}$ Reduction. Angew. Chem. Int. Ed. 2016, 55, 5789-5792.

115. Iyengar, P.; Huang, J.; De Gregorio, G. L.; Gadiyar, C.; Buonsanti, R., Size Dependent Selectivity of Cu Nano-Octahedra Catalysts for the Electrochemical Reduction of $\mathrm{CO}_{2}$ to $\mathrm{CH}_{4}$. Chem. Comm. 2019, 55, 8796-8799.

116. Ghosh, S.; Manna, L., The Many “Facets" of Halide Ions in the Chemistry of Colloidal Inorganic Nanocrystals. Chem. Rev. 2018, 118, 7804-7864.

117. Guo, H.; Chen, Y.; Cortie, M. B.; Liu, X.; Xie, Q.; Wang, X.; Peng, D.-L., Shape-Selective Formation of Monodisperse Copper Nanospheres and Nanocubes via Disproportionation Reaction Route and Their Optical Properties. J. Phys. Chem. C 2014, 118, 9801-9808. 